PERSPECTIVA TEOLÓGICA ADERE A UMA LICENÇA CREATIVE COMMONS ATRIBUIÇÃO 4.0 INTERNACIONAL - (CC BY 4.0 )

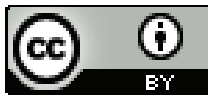

DOI: $10.20911 / 21768757 v 53 n 1 p 119 / 2021$

\title{
DE LA AMORIS LAETITIA A LA FRATELLI TUTTI: LA IMPORTANCIA DE LAS RELACIONES FAMILIARES
}

From Amoris Laetitia to Fratelli Tutti: the Importance of Family Relationships

Martín Carbajo Núñez *

SUMARIO: A la luz la exhortación post-sinodal Amoris Laetitia, el presente artículo estudia "el evangelio de la familia" y la importancia de las relaciones familiares en la vida social. Hoy se siente la necesidad "de una robusta inyección de espíritu familiar" (AL, n. 183). La encíclica Fratelli tutti recoge esa necesidad y aboga por favorecer la fraternidad y la amistad social en nuestra sociedad globalizada que "nos hace más cercanos, pero no más hermanos". La experiencia de familia es fundamental en el desarrollo de las cuatro relaciones fundamentales del ser humano ( $1^{\text {a }}$ parte) y en la educación ( $2^{\underline{a}}$ parte). Además, la familia introduce la fraternidad en el mundo ( $3^{\underline{a}}$ parte) y es el agente principal de la ecología integral ( $4^{\underline{a}}$ parte).

PALABRAS CLAVE: Familia. Matrimonio. Fraternidad, Amistad social. Ecología integral.

ABSTRACT: In the light of the post-synodal exhortation Amoris Laetitia, this article studies "the gospel of the family" and the importance of family relationships in our social life. Today, there is an "omnipresent need for a healthy injection of family spirit" (AL 183). The encyclical Fratelli tutti reflects this need and proposes to promote fraternity and social friendship in a globalized society that "makes us neighbors but does not make us brothers". The article argues that the family is essential for developing our four fundamental relationships $\left(1^{\text {st }}\right.$ part $)$ as well as for education $\left(2^{\text {nd }}\right.$ part). Furthermore, the family introduces fraternity into the world $\left(3^{\text {rd }}\right.$ part) and is the principal agent of an integral ecology $\left(4^{\text {th }}\right.$ part $)$.

KEYWORDS: Family. Marriage. Fraternity. Social Friendship. Integral Ecology.

* Pontificia Universidad Antonianum, Roma, Itália. 


\section{Introducción}

la luz de la exhortación post-sinodal Amoris Laetitia (AL) ${ }^{1}$, el presente Aartículo estudia "el evangelio de la familia" y la importancia de las relaciones familiares en la vida social. El Papa Francisco afirma que hoy, por todos los lados, se siente la necesidad "de una robusta inyección de espíritu familiar" (AL, n. 183). La encíclica Fratelli tutti (FT) ${ }^{3}$ recoge esa necesidad y aboga por favorecer la fraternidad y la amistad social en nuestra sociedad tecnológica y globalizada que "nos hace más cercanos, pero no más hermanos" (CV, n. 19) .

AL invita al cuidado pastoral de la familia, en cuanto célula vital de la sociedad $^{5}$ y resalta que este cuidado tiene una gran importancia para todo el cuerpo social y para el desarrollo armonioso de sus miembros.

AL la entiendo como una propuesta para las familias cristianas, que las estimule a valorar los dones del matrimonio y de la familia, y a sostener un amor fuerte [...]. Procura alentar a todos para que sean signos de misericordia y cercanía allí donde la vida familiar no se realiza perfectamente o no se desarrolla con paz y gozo (AL, n. 5).

La lectura de AL debería ayudar a que cada uno "se sienta llamado a cuidar con amor la vida de las familias" (AL, n.7) y a tener misericordia de la fragilidad humana ${ }^{6}$. Por tanto, esta exhortación afirma la importancia de la relación y del cuidado amoroso por encima del rígido cumplimiento de normas, incluso cuando se "corra el riesgo de mancharse con el barro del camino"7.

\footnotetext{
${ }^{1}$ FRANCISCO, Papa. Exhortación apostólica postsinodal Amoris Laetitia. Acta Apostolicae Sedis (AAS), Vaticano, v. 108, n. 4, p. 311-446, 2016a. AL recoge la reflexión de dos Sínodos sobe la familia, celebrados en 2014 y 2015, a saber: la III Asamblea General Extraordinaria del Sínodo de los Obispos, sobre "los desafíos pastorales de la familia en el contexto de la evangelización" (5-19.10.2014); y la XIV Asamblea General ordinaria del Sínodo de los obispos (4-25.10.2015), sobre "la vocación y la misión de la familia en la Iglesia y en el mundo contemporáneo".

2 "Se trata de hacer experimentar que el Evangelio de la familia es alegría que llena el corazón y la vida entera" (AL, n. 200). Cf. KASPER, W. El evangelio de la familia. Santander: Sal Terrae, 2014; TEJERO, E. El evangelio de la casa y de la familia. Pamplona: Eunsa, 2014.

${ }^{3}$ FRANCISCO, Papa . Carta encíclica Fratelli tutti. Ciudad del Vaticano: LEV, 2020a.

${ }^{4}$ BENEDICTO XVI, Papa. Carta encíclica Caritas in veritate [CV]. AAS, Vaticano, v. 101, p. 641-709, 2009a.

5 "La familia es un bien del cual la sociedad no puede prescindir" (AL, n. 44), uno de "los valores no negociables". BENEDICTO XVI, Papa. Exhortación apostólica postsinodal Sacramentum Caritatis. AAS, Vaticano, v. 99, n. 3, p. 105-180, 2007.

6 "Hay que acompañar con misericordia y paciencia, [...] más allá de sus defectos y caídas". FRANCISCO, Papa. Exhortación apostólica Evangelii gaudium [EG]. AAS, Vaticano, v. 105, n. 44, p. 1019-1137, 2013b; "Asumir la lógica de la compasión con los frágiles" AL, n. 308; cf. BONFRATE, G.; YÁÑ̃Z, M. Amoris Laetitia, la Sapienza dell'amore: Fragilità e belleza della relazione nel matrimonio e nella famiglia. Roma: Studium, 2017; CAPANTINI, L.; GRONCHI, M. La vulnerabilità. Cinisello Balsamo: San Paolo, 2018.

${ }^{7}$ AL, n. 308. La relación humana tiene prioridad sobre la norma. También en el Antiguo Testamento "el decálogo adquiere su verdad más profunda" en la relación de confianza que Dios ha instaurado con su pueblo, al que invita a dejarse abrazar por su misericordia. FRANCISCO, Papa. Carta encíclica Lumen Fidei. AAS, Vaticano, v. 105, n. 43, p. 555-596, 2013a.
} 
En diversos documentos, el Papa Francisco ha desarrollado ulteriormente esta perspectiva relacional que puede colmar al ser humano de la alegría del Espíritu Santo. En efecto, la iniciativa amorosa de $\operatorname{Dios}^{8}$ nos lleva a la alegría del anuncio evangélico (EG, n.236), a la vivencia gozosa del amor en familia (AL), a la alegría de la verdad ${ }^{9}$ y a exultar de gozo por haber sido llamados a la santidad ${ }^{10}$.

Las últimas encíclicas del Papa Francisco desarrollan también el tema de las relaciones familiares y fraternas desde perspectivas complementarias. La familia humana que presenta FT forma parte de la familia cósmica que promueve la encíclica Laudato si' $[\mathrm{LS}]^{11}$, pues en la casa común todos estamos relacionados (FT) y "todo está relacionado" (LS). Las dos encíclicas nos invitan a escuchar el clamor de la tierra y el grito de los pobres (LS, n. 38), si bien la LS se focaliza en el primero y FT en el segundo. Ambos gritos son inseparables y no se puede escuchar uno sin atender al otro.

La primera parte de este artículo aclara el concepto de familia y resalta su importancia en el desarrollo de las cuatro relaciones fundamentales del ser humano: con Dios, consigo mismo, con los demás y con la creación. El Dios trinitario es una familia y el ser humano, creado a su imagen, es un ser familiar que desarrolla su intrínseca sociabilidad en el encuentro afectuoso con los demás miembros de la sociedad y con la entera creación. La segunda parte presenta a la familia como nuestra primera escuela y resalta la importancia del aspecto relacional en la educación universitaria. A continuación, el artículo muestra que la familia introduce la fraternidad en el mundo ( $3^{\mathrm{a}}$ parte) y que ella es el agente principal de la ecología integral ( $4^{a}$ parte).

\section{El concepto de familia y sus cuatro niveles}

El "pequeño núcleo familiar no debería aislarse de la familia ampliada" (AL, n. 187). Acogiendo esta invitación, usaremos el concepto de familia en cuatro niveles estrechamente relacionados: espiritual, personal, social y natural; es decir, la familia de los creyentes en Dios Padre ${ }^{12}$, la familia

\footnotetext{
8 “No se comienza a ser cristiano por una decisión ética o una gran idea, sino por el encuentro con un acontecimiento, con una Persona, que da un nuevo horizonte a la vida". BENEDICTO XVI, Papa. Carta encíclica Deus caritas est [DC]. AAS, Vaticano, v. 98, n. 1, p. 217-252, 2006. ${ }^{9}$ FRANCISCO, Papa. Constitución Apostólica Veritatis gaudium [VG]. Regno-Documenti, Vaticano, v. 63, n. 5, p. 137-165, 2018 a.

${ }^{10}$ FRANCISCO, Papa. Exhortación apostólica Gaudete et exsultate [GE]. Ciudad del Vaticano: LEV, 2018b.

${ }^{11}$ FRANCISCO, Papa. Carta encíclica Laudato si'. AAS, Vaticano, v. 107, n. 9, p. 847-945, 2015a.

${ }^{12}$ La Iglesia es la gran familia de los Hijos de Dios: "existe como fermento y alma de la sociedad humana, que debe ser renovada en Cristo y transformada en familia de Dios" (CONCILIO VATICANO II. Constitución pastoral Gaudium et spes. AAS, Vaticano, v. 58, p. 1025-1120, 1966); cf. Catecismo de la Iglesia Católica [CCC], n. 854. Ciudad del Vaticano: LEV, 1997; JUAN PABLO II, Papa. Exhortación apostólica Familiaris consortio. AAS, Vaticano,
} 
nuclear, normalmente basada en el matrimonio, la familia humana universal y la familia cósmica.

El concepto de familia ayuda a entender la relación cercana que existe entre todos los seres ("Todo está relacionado", LS, n. 92). Ayuda también a expandir el concepto de solidaridad, de modo que la búsqueda de la igualdad vaya acompañada del respeto a la diversidad ${ }^{13}$. Somos diferentes, pero hermanos, miembros de la única familia cósmica y habitantes de la misma casa.

\subsection{Comunión trinitaria y comunión familiar}

Dios es una familia, "puesto que lleva en sí mismo paternidad, filiación y la esencia de la familia que es el amor" (JUAN PABLO II, 1979, p. 182) ${ }^{14}$. En efecto, el Dios trinitario (tri-unidad) es comunión en la pluralidad ${ }^{15}$, fuente de toda unidad y de toda diferencia ${ }^{16}$. El Padre es el Amante, el Hijo el Amado, el Espíritu es el Amor. Todo ha sido creado conforme a ese modelo divino y, por tanto, "lleva en sí una estructura propiamente trinitaria" (LS, n. 239) ${ }^{17}$.

Existe una cierta analogía entre la comunión trinitaria y la comunión que se vive en el matrimonio. "El Dios Trinidad es comunión de amor, y la familia es su reflejo viviente" (AL, n. 11); es decir, "la familia es imagen de Dios, que es comunión de personas" (AL, n. 71).

La paternidad y maternidad humanas, aun siendo biológicamente parecidas a las de otros seres de la naturaleza, tienen en sí mismas, de manera esencial y exclusiva, una semejanza con Dios, sobre la que se funda la familia, entendida como comunidad de vida humana, como comunidad de personas unidas en el amor (communio personarum). [...] La familia, que se inicia con el amor del

\footnotetext{
v. 74, p. 81-191, 1982. La fe "nos da una familia, la familia universal de Dios en la Iglesia Católica" (BENEDICTO XVI, Papa. Discurso inaugural, Aparecida, 13.05.2007. In: CELAM. $V$ Conferencia General del Episcopado Latinoamericano y del Caribe, Documento conclusivo. Bogotá: Paulinas, 2008a).

${ }^{13}$ FLORES, N. M. "Our sister, mother earth": solidarity and familial ecology in Laudato si'. Journal of Religious Ethics, New Jersey, v. 46, n. 3, p. 463-478, 2018.

${ }^{14}$ JUAN PABLO II, Papa. Homilía en Puebla de los Ángeles, 28.01.1979. In: JUAN PABLO II, Papa. Insegnamenti di Giovanni Paolo II. Ciudad del Vaticano: LEV, 1979. p. 180-185.

${ }_{15}$ 1Jn 4,8. Cf. AUGUSTINUS. De Trinitate, XV, c. 16. In: Migne, J.-P. (Ed.). Patrologia Latina [PL]. Paris: 1841-1864. v. 42.

${ }^{16}$ La unidad divina "será tanto más evidente cuanto más clara sea la distinción de las personas, y viceversa". En efecto, la total comunión de las tres personas "no excluye, sino que afirma, la existencia de tres 'yo', pero al mismo tiempo elimina radicalmente la posibilidad de un 'tuyo' y un 'mío". Esa unidad se expresa en "la mutua inhabitación o perichóresis de las mismas" (LADARIA, L. F. La Trinidad, Misterio de Comunión. Salamanca: Secretariado Trinitario, 2002. p. 128-129).

17 "Creatura mundi est quasi quidem liber, in quo relucet et repraesentatur el legitur Trinitas fabricatrix" (BUENAVENTURA, Breviloquium, II c.12 (Quaracchi V 230a)).
} 
hombre y la mujer, surge radicalmente del misterio de Dios (JUAN PABLO II, 1994a, n. 6-8) ${ }^{18}$.

Esta analogía se encuentra en la Sagrada Escritura y en la Tradición, que "nos revelan la Trinidad con características familiares". Además, Cristo "elevó el matrimonio a signo sacramental de su amor por la Iglesia" (AL, n. 71 y 73).

\subsection{El hombre es un ser familiar}

La persona humana es un ser familiar, creado a imagen del Dios trinitario (Gen 1,27). Relacionándose, desarrolla su propia identidad, pues "asume en su propia existencia ese dinamismo trinitario que Dios ha impreso en ella" (LS, n. 240).

La diferencia sexual expresa la vocación del ser humano a la unidad en la diversidad. Asimismo, la "sociedad de hombre y mujer es la expresión primera" de su intrínseca sociabilidad (GS, n. 12).

\subsubsection{Necesitamos superar la actual antropología negativa}

La Modernidad ha asumido una concepción antropológica negativa (homo homini lupus ${ }^{19}$ ) que reduce la persona a homo oeconomicus, egocéntrico, utilitarista, radicalmente auto-interesado y, por tanto, predecible e incapaz de gratuidad. El hombre no sería de fiar, pues su actuar respondería siempre al instinto de autoconservación ${ }^{20}$.

El dualismo antropológico ha acentuado la separación materia/espíritu y ha llevado a despreciar el cuerpo y a reducir el valor del matrimonio a la unión espiritual. AL recuerda, sin embargo, que "un amor sin placer ni pasión no es suficiente para simbolizar la unión del corazón humano con Dios"' (AL, n. 142).

El Concilio Vaticano II supera ese dualismo y afirma que el ser humano es "corpore et anima unus" (GS, n. 14). El Verbo encarnado no nos redime de la materia, sino con la materia y con el cuerpo. Al mismo tiempo, el hombre es un ser dialógico, social, que "no puede vivir ni desplegar sus cualidades sin relacionarse con los demás" (GS, n. 12). En él permanece siempre vivo "el deseo de familia" (AL, n. 1).

\footnotetext{
${ }^{18}$ JUAN PABLO II, Papa. Carta a las familias Gratissimam sane. AAS, Vaticano, v. 86, p. 868-925, 1994a.

${ }_{19}$ Afirmación de Plauto (Asinaria, acto II) recogida por HOBBES, T. The Leviathan. New York: Crooke, 1651. p. 98-102.

${ }^{20}$ Este sería el principio básico de la civilización occidental (ADORNO T. W.; HORKHEIMER M. Dialectic of Enlightenment. New York: Herder \& Herder, 1997. p. 29).
} 
Para entrar serenamente en relación, el ser humano necesita cuidar también de sí mismo, madurar "su manera de relacionarse" (n. 239), atesorar "una riqueza interior que se alimenta en la lectura, la reflexión personal, la oración y la apertura a la sociedad" (AL, 141) ${ }^{21}$. La capacidad de escucharse, discernir y articular la propia historia hace posible el encuentro sereno y enriquecedor con el otro.

\subsubsection{La familia es el hábitat natural del ser humano}

La familia es el hábitat natural del ser humano; es decir, el ambiente más adecuado para que desarrolle su identidad y su sociabilidad. "La presencia clara y bien definida de ambas figuras, femenina y masculina, crea el entorno más adecuado para el crecimiento del niño" (AL, n. 175), le ayuda a ser él mismo, sin máscara ( $\mathrm{AL}, \mathrm{n} .315)^{22}$ y le inserta como miembro activo en el proyecto común.

"El don recíproco de sí por parte del hombre y de la mujer crea un ambiente de vida en el cual el niño puede nacer y desarrollar sus potencialidades" (CA, n. 39), pues allí encuentra "el núcleo afectivo primordial que le da consistencia y seguridad" (JUAN PABLO II, 1996, p. 231) ${ }^{23}$, además de protegerle de influencias indeseables (violencia, pornografía).

En la familia,

el hombre recibe las primeras nociones sobre la verdad y el bien; aprende qué quiere decir amar y ser amado. [...] Hay que volver a considerar la familia como el santuario de la vida. En efecto, es sagrada: es el ámbito donde la vida, don de Dios, puede ser acogida y protegida de manera adecuada (CA, n. 39) ${ }^{24}$.

Sintiéndose querido por sí mismo, como único e irrepetible, el niño desarrolla su propia identidad y se prepara para ser un miembro activo de la familia de los Hijos de Dios, de la familia humana universal y de la gran familia cósmica. Las familias que vive gozosamente su cristianismo (AL, n. 86) tienen ese poder de atracción que hace crecer a la Iglesia ${ }^{25}$ y son los "principales sujetos de la pastoral familiar" (AL, n. 200).

\footnotetext{
${ }^{21}$ Cf. SALONIA, G. Amoris Laetitia: Dalla sapienza evangelica percorsi di crescita nelle relazioni familiari. In: BONFRATE, G.; YÁÑEZ, H. M. (Ed.). Amoris Laetitia, la sapienza dell'amore: Fragilità e bellezza della relazione nel matrimonio e nella famiglia. Roma: Studium, 2017. p. 168-182. Aquí p. 178.

22 "Hacer crecer es ayudar al otro a moldearse en su propia identidad" (AL, n. 221).

${ }^{23}$ JUAN PABLO II, Papa. Ángelus 30.01.1994. In: JUAN PABLO II, Papa. Insegnamenti di Giovanni Paolo II. Ciudad del Vaticano: LEB, 1996. v. XVII/1, p. 231-234.

${ }^{24}$ JUAN PABLO II, Papa. Carta encíclica Centesimus annus [CA]. AAS, Vaticano, v. 83, p. 793-867, 1981.

25 "La Iglesia no crece por proselitismo sino por atracción" (EG, n. 14). De hecho, la familia tiene un papel decisivo en la evangelización (cf. FC, n. 71).
} 


\subsubsection{Ayudar al discernimiento}

La persona necesita crecer como ser fraterno y familiar, pero también autónomo, activo y responsable. AL invita a los pastores a que dejen espacio a la conciencia de los fieles y a que les ayuden a desarrollar su propio discernimiento, pues "estamos llamados a formar las conciencias, pero no a pretender sustituirlas" (AL, n. 37$)^{26}$. De hecho, resulta muy significativo que AL reconduzca al ámbito de la conciencia personal la búsqueda de una salida a ciertas situaciones irregulares de la familia ${ }^{27}$.

“Es mezquino detenerse sólo a considerar si el obrar de una persona responde o no a una ley o norma general, porque eso no basta para discernir y asegurar una plena fidelidad a Dios en la existencia concreta de un ser humano" ( $\mathrm{AL}$, n. 304).

$A L$ invita "a un responsable discernimiento personal y pastoral" (AL, $\mathrm{n}$. $300)^{28}$, aunque reconoce que a algunos le resultaría más cómodo impulsar una pastoral rígida "que no dé lugar a confusión alguna" (AL, n. 308). Necesitamos "un discernimiento pastoral cargado de amor misericordioso, que siempre se inclina a comprender, a perdonar, a acompanar, a esperar, y sobre todo a integrar" (AL, n. 312).

Hay que evitar "una fría moral de escritorio" ( $A L, n$. 312) que emite juicios sin tener en cuenta "la complejidad de las diversas situaciones" (AL, n. 296) y sin un adecuado discernimiento (AL, n. 298). Por desgracia, a veces "nos comportamos como controladores de la gracia y no como facilitadores" (AL, n. 310).

El discernimiento tiene el objetivo de identificar el bien posible para el sujeto en situaciones concretas; es decir, trata de encontrar "aquello que, por ahora, es la respuesta generosa que se puede ofrecer a Dios, y descubrir con cierta seguridad moral que esa es la entrega que Dios mismo está reclamando" $(\mathrm{AL}, \mathrm{n} .303)^{29}$.

${ }^{26}$ Los pastores "están obligados a discernir bien las situaciones" (FC, n. 84).

27 "Nos cuesta dejar espacio a la conciencia de los fieles, que muchas veces [...] pueden desarrollar su propio discernimiento ante situaciones donde se rompen todos los esquemas" (AL, n. 37).

${ }^{28}$ cf. EG, n. 33; 50; 154.

${ }^{29}$ CARBAJO NÚÑEZ, M. Generación híper-conectada y discernimiento: Una perspectiva franciscana. Selecciones de Franciscanismo, Murcia, v. 138, p. 227-244, 2018a. "In un certo momento la diagnosi, il problem solving e l'analisi morale trovano la soluzione". Invece, "il presupposto fondamentale del discernimento è che esso non riguarda un problema, ma piuttosto una vita in camino [... e cerca di] identificare dove e come Dio sta invitando quella persona o quella comunità alla conversione e alla vita" (SPADARO; CAMELI; 2016, p. 7-8); cf. Majorano, S., The Alphonsian criteria of merciful pastoral discernment in the light of chapter VIII of the exhortation Amoris Laetitia. Studia Moralia, Roma, v. 55, n. 1, p. 7-32, 2017. 


\subsection{La anhelada familia universal}

La sociedad está llamada a ser una gran familia. Sin embargo, "nuestras sociedades se caracterizan por un creciente individualismo" (FRANCISCO, 2014a, p. 8) que debilita los vínculos interpersonales y "desnaturaliza los vínculos familiares" (EG, n. 67). Esto "lleva a encerrarse en un pequeño nido de seguridad y a sentir a los otros como un peligro molesto" (AL, n. 187). De hecho, la familia nuclear "atraviesa una crisis cultural profunda" (EG, n. 66).

Los medios de comunicación y las redes sociales transmiten sólo una ilusión de comunidad, mientras responden a la lógica del consumismo. Curiosamente, los usuarios más frecuentes de las nuevas tecnologías de la comunicación tienden a ser los menos empáticos ${ }^{30}$. Necesitamos restablecer los bienes relacionales y las relaciones familiares a todos los niveles, para así poder superar la dialéctica de perenne conflicto.

\subsection{Una familia cósmica}

La entera creación es una gran familia, un sistema vital de relaciones, un espacio de encuentro y de reciprocidad. En efecto, "todos los seres del universo estamos unidos por lazos invisibles y conformamos una especie de familia" (LS, n. 89). En ese hogar común, Dios se manifiesta y entra en diálogo con nosotros. "Toda la creación, en definitiva, está pensada para crear el lugar de encuentro entre Dios y su criatura" (BENEDICTO XVI, 2008b, p. 6).

La entera realidad es dialógica, una gran familia que vive en la misma casa $^{31}$. Cada criatura "debe ser valorada con afecto y admiración", pues "todos los seres nos necesitamos unos a otros" (LS, n. 42).

\section{Educación y relaciones familiares}

La familia, en cuanto "primera escuela de relaciones sociales" (JUAN PABLO II, 1996, p. 231), debe potenciar la intrínseca sociabilidad del ser humano, enseñando "con paciencia a los hijos a tratarse como hermanos" (AL, n. 195). "El vínculo de fraternidad que se forma en la familia entre los hijos, si se da en un clima de educación abierto a los demás, es una gran

\footnotetext{
${ }^{30}$ On the rapid decline of empathy among university students, especially from 2001 onwards: KONRATH S.; O'BRIEN, E.H.; HSING, C. Changes in Dispositional Empathy in American College Students over Time: A Meta-analysis. Personality and Social Psychology Review, Thousand Oaks, v. 15, n. 2, p. 180-198, 2011.

${ }^{31}$ MCFAGUE, S. The body of God: An ecological theology. Minneapolis: Fortress, 1993. p. 103-129.
} 
escuela de libertad y de paz. En la familia, entre hermanos, se aprende la convivencia humana" (AL, n. 194).

Los padres que "privan al hijo único de la posibilidad de otros hermanos" le están privando "de la experiencia de la fraternidad". "Otra situación anómala es la de aquellas familias que no valoran el lugar fundamental que ocupan los ancianos" (CONFERENCIA EPISCOPAL ESPAÑOLA, 2001, n. 82). El Papa Francisco ha recordado que "los ancianos tienen la sabiduría de un pueblo" (FRANCISCO, 2018c, p. 8) y que los niños "traen vida, alegría, esperanza" (FRANCISCO, 2018d, p. 8).

Por desgracia, hoy se promueve una mentalidad antinatalista que está también favorecida por las biotecnologías, el consumismo, "la industrialización, la revolución sexual, el miedo a la superpoblación, los problemas económicos" (AL, n. 42).

\subsection{La familia es la más importante escuela}

La familia humana tiene el derecho primario a educar ${ }^{32}$ y "es la primera y más importante escuela" (FRANCISCO, 2016b, p. 4). En ella se experimenta y se aprende la misericordia, que es "la viga maestra que sostiene la vida de la Iglesia" (AL, n. 310). Se aprenden también los valores humanos (AL, n. 274) y las "virtudes sociales, como el respeto de las personas, la gratuidad, la confianza, la responsabilidad, la solidaridad, la cooperación" (BENEDICTO XVI, 2012, p. 695) ${ }^{33}$. Asimismo, la familia es la base para defenderse "de tantas colonizaciones, como la del dinero o de las ideolo-

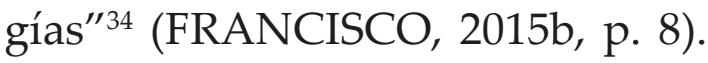

La Iglesia y los entes sociales deben "sostener y colaborar con las familias" en esa tarea educativa (FRANCISCO, 2020b, p. 4). En este sentido, la escuela católica desempeña una labor fundamental (AL, n. 279).

Necesitamos poner en práctica el tipo de relaciones misericordiosas que, normalmente, aprendemos de pequeños en la casa familiar y que nos ayudan a "descubrir el rostro amoroso de Dios" (FRANCISCO, 2016b, p. 4-5) y el misterio del "nosotros trinitario" (JUAN PABLO II, 1994a, p. 877) ${ }^{35}$. De ello depende el futuro de la gran familia cósmica y "el desarrollo integral de todo hombre y de todos los hombres" (PP, n. 14) ${ }^{36}$.

32 FRANCISCO, Papa. Discurso al cuerpo diplomático acreditado ante la Santa Sede, 9.01.2020. L'Osservatore Romano, Vaticano, n. 6, p. 4-5, 10 ene. 2020. Aquí p. 4.

${ }^{33}$ La familia es "el ámbito de la socialización primaria, porque es el primer lugar donde se aprende a colocarse frente al otro, a escuchar, a compartir, a soportar, a respetar, a ayudar, a convivir" (AL, n. 276).

${ }^{34}$ Entre esas ideologías, el Papa habla de la ideología de género ( $\left.\mathrm{AL}, \mathrm{n} .56\right)$, que debe ser contrastada con una buena educación sexual en la familia (AL, n. 280-286).

${ }^{35}$ La familia, "iglesia doméstica", exige sacrificio, tolerancia, perdón y reconciliación (cf. FC 21).

${ }^{36}$ PABLO VI, Papa. Carta encíclica Populorum progressio [PP]. AAS, Vaticano, v. 59, p. 257299, 1967. 


\subsection{Relaciones familiares en la universidad}

Las relaciones familiares deben tener un lugar privilegiado también en la enseñanza universitaria ${ }^{37}$. En vez de limitarse a transmitir conocimientos en modo meramente instrumental, la universidad debe reforzar los lazos comunitarios y la visión holística de la realidad. También a nivel interno, "una educación fructífera no depende fundamentalmente ni de la preparación del profesor ni de las competencias de los alumnos; depende más bien de la calidad de la relación que se establece entre ellos" (CONGREGACIÓN PARA LA EDUCACIÓN CATÓLICA, 2020).

La búsqueda de la unidad en la diversidad, típica de la vida familiar auténtica ${ }^{38}$, debe continuar en la educación universitaria. "El modelo es el poliedro, que refleja la confluencia de todas las parcialidades que en él conservan su originalidad" (EG, n.236). Este enfoque multidimensional requiere "construir una aldea de la educación donde se comparta en la diversidad el compromiso por generar una red de relaciones humanas y abiertas" (FRANCISCO, 2019, p. 8) ${ }^{39}$. Además, busca "superar fragmentaciones y contraposiciones y reconstruir el tejido de las relaciones" (FRANCISCO, 2019, p. 8).

También dentro de la Iglesia "es necesaria una unidad de doctrina y de praxis, pero ello no impide que subsistan diferentes maneras de interpretar algunos aspectos de la doctrina o algunas consecuencias que se derivan de ella" (AL, n. 3).

\section{Amor familiar y amor social}

Más que ofrecer una doctrina sobre la familia, AL anima a vivir el amor familiar y a hacer de la familia el lugar donde cada uno crece en el amor ${ }^{40}$, a través de un proceso "dinámico de desarrollo y realización" (AL, n. 37).

Con frecuencia presentamos el matrimonio de tal manera que su fin unitivo, el llamado a crecer en el amor y el ideal de ayuda mutua, quedó opacado por un acento casi excluyente en el deber de la procreación (AL, n. 36).

\footnotetext{
${ }^{37}$ Hemos desarrollado más ampliamente este tema en nuestro artículo: CARBAJO NÚÑEZ, M. Education and integral ecology: The role of family, spirituality, and university. Carthaginensia, Murcia, v. 37, n. 71, 2021a.

${ }^{38} \mathrm{AL}, \mathrm{n}$. 139. "La unidad a la que hay que aspirar no es uniformidad, sino una unidad en la diversidad [...]. Hace falta liberarse de la obligación de ser iguales" Ibid.

39 "Unità e differenza non si escludono, anzi si implicano. In caso contrario, saremmo di fronte a un'unità soffocante" (Pacto Educativo Global 3.1).

${ }^{40} \mathrm{El}$ amor conyugal es el centro del matrimonio (GS, n. 47-52). "La gracia del sacramento del matrimonio está destinada ante todo a perfeccionar el amor de los cónyuges" (AL, n. 89; CCC, n. 1641).
} 
Esas relaciones gratuitas y desinteresadas que se viven en la familia parental deben también alargarse al ámbito público. La modernidad, sin embargo, ha relegado las relaciones amistosas al ámbito privado, pues considera que esas dependencias afectivas entorpecen el correcto funcionamiento del engranaje público.

\section{Contradiciendo esa mentalidad, la encíclica FT promueve}

la fraternidad y la amistad social". Uniendo ambas expresiones en el mismo título, el Papa presenta la amistad como una categoría no sólo privada, sino también social; es decir, incluye las relaciones familiares en el núcleo de vida pública. En efecto, "la caridad está en el corazón de toda vida social sana y abierta (FT, n. 184$)^{41 .}$

AL añade que "es precisamente la familia la que introduce la fraternidad en el mundo" pues allí tenemos "la primera experiencia de hermandad" (AL, n. 194).

\subsection{El deseo de amor verdadero}

Le exhortación $A L$ invita a acompañar y potenciar el "deseo de amor verdadero" (AL, n. 294) que anida en el corazón humano y que se manifiesta de manera particular en la vida conyugal y familiar. Esto supone pasar "de la lógica legalista del mínimo indispensable a la lógica del máximo posible" (COSTA, 2016, p. 362, trad. mía) ${ }^{42}$.

La Iglesia entera ${ }^{43}$ intenta acompañar y promover esa vocación al amor que está a la base de toda su pastoral ${ }^{44}$. Por eso, "la pastoral prematrimonial y la pastoral matrimonial deben ser ante todo una pastoral del vínculo, donde se aporten elementos que ayuden tanto a madurar el amor como a superar los momentos duros" (AL, n. 211) ${ }^{45}$. Necesitamos aprender a dialogar (AL, n. 136-141), a decir "permiso, gracias, perdón" $(\mathrm{AL}, \mathrm{n} .133)^{46}$.

${ }^{41}$ Hemos desarrollado este tema en: CARBAJO NÚÑEZ, M. La fraternità nell'enciclica Fratelli tutti: Radici francescane. Collectanea Franciscana, Roma, v. 90, 2021b.

${ }^{42}$ Pertanto, "il discernimento si rivela persino più esigente della norma". Ibid.

${ }^{43} \mathrm{AL}, 229$. Los "fieles que están viviendo situaciones complejas" necesitan ser acompañados en su discernimiento no sólo por los pastores, sino también por "laicos que viven entregados al Señor" (AL, n. 312).

44 "Hay que preparar a los jóvenes para el matrimonio, hay que enseñarles el amor. El amor no es cosa que se aprenda, iy sin embargo no hay nada que sea más necesario enseñar! [...] Éste es uno de los temas fundamentales sobre el que centré mi sacerdocio" (JUAN PABLO II, Papa. Cruzando el umbral de la esperanza. Barcelona: Plaza \& Janés, 1994b. p. 132) cf. FC, 11; CID VÁZQUEZ, M.T. El amor como hilo conductor de la pastoral familiar en Amoris Laetitia. Familia, Salamanca, v. 54, p. 69-84, 2017.

${ }^{45}$ Una "espiritualidad del vínculo" (AL, n. 315).

${ }^{46}$ Decir "por favor" (AL, n. 266). 
También la encíclica FT organiza todo el discurso en torno al amor fraterno. Benedicto XVI había ya insistido en que "la caridad es la vía maestra de la doctrina social de la Iglesia" $(\mathrm{CV}, \mathrm{n}$. 2) y de ella emanan todas sus indicaciones. $A L$ insiste en su importancia para la sociedad.

El amor social, reflejo de la Trinidad, es en realidad lo que unifica el sentido espiritual de la familia y su misión fuera de sí, porque hace presente el kerygma con todas sus exigencias comunitarias. La familia vive su espiritualidad propia siendo al mismo tiempo una iglesia doméstica y una célula vital para transformar el mundo (AL, n. 324).

Por desgracia, hoy predomina la cultura de lo provisorio, que lleva a pasar rápidamente de una relación afectiva a otra, tal como sucede con los contactos de las redes sociales: "todo es descartable" (AL, n. 39). Esto se traduce en una perenne inseguridad y en un aumento de los divorcios.

\subsection{Amor social}

El amor no es sólo el principio de las micro-relaciones en el ámbito privado, "sino también de las relaciones sociales, económicas y políticas" (CV, n. 2). De hecho, en la vida pública, el amor es la potencialidad humana que más influye en la sociedad.

La energía principal que mueve al alma humana es el amor. La naturaleza humana, en su esencia más profunda, consiste en amar. En definitiva, a cada ser humano se le encomienda una sola tarea: aprender a querer, a amar de modo sincero, auténtico y gratuito (GUILHERMO DE SAINT-THIERRY apud BENEDICTO XVI, 2009b, p. 1).

En lugar de encerrarse en sí misma, la familia nuclear debe acoger y salir hacia los demás, "para derramar su bien en otros" (AL, n. 234), convirtiéndose así en "un nexo de integración de la persona con la sociedad y en un punto de unión entre lo público y lo privado" (AL, n. 181).

Al hablar de amor social, el Papa da prioridad al cultivo de las relaciones personales sobre la eficiencia en conseguir objetivos materiales. También la pastoral eclesial debe centrarse no tanto en programas y actividades cuanto en acompañar personas y en generar procesos ${ }^{47}$. Tendrá en cuenta que la vida familiar es poliédrica y dinámica, "un camino de permanente crecimiento" (AL, n. 37) y de "constante maduración" (AL, n. 134)

Las relaciones familiares son también imprescindibles en el ámbito económico ${ }^{49}$, pues favorecen la colaboración y la promoción desinteresada

\footnotetext{
${ }^{47}$ AL, n. 261. “Un proceso dinámico, que avanza gradualmente" (FC, n. 9). Sacerdotes, religiosos y laicos deben iniciarse en "el arte del acompañamiento" (EG 169).

${ }^{48}$ La ley de la gradualidad, que "posibilita una profundización gradual de las exigencias del Evangelio" no debe ser confundida con la "gradualidad de la ley" (AL, n. 295).

${ }^{49}$ Cf. CARBAJO NÚÑEZ, M. Franziskanische Wirtschaft: Ein Vorschlag um aus der Krise Herauszukommen. Heiligenkreuz: Be\&Be Verlag, 2021c.
} 
de cada uno de sus miembros. El amor es la fuerza principal y el destino del desarrollo humano integral. En efecto, "el principio de gratuidad y la lógica del don, como expresiones de fraternidad, pueden y deben tener espacio en la actividad económica ordinaria" (CV, n. 36).

\section{Familia y ecología}

La exhortación Amoris Laetitia afirma que la familia parental es el agente principal de la ecología integral, "porque es el sujeto social primario, que contiene en su seno los dos principios-base de la civilización humana sobre la tierra: el principio de comunión y el principio de fecundidad" (AL, n. 277).

Una vida familiar sana ayuda a superar el individualismo y a restablecer nuestras cuatro relaciones fundamentales, haciendo posible la ecología integral.

En la familia se cultivan los primeros hábitos de amor y cuidado de la vida, como por ejemplo el uso correcto de las cosas, el orden y la limpieza, el respeto al ecosistema local y la protección de todos los seres creados. La familia es el lugar de la formación integral, donde se desenvuelven los distintos aspectos, íntimamente relacionados entre sí, de la maduración personal. [...] Estos pequeños gestos de sincera cortesía ayudan a construir una cultura de la vida compartida y del respeto a lo que nos rodea (LS, n. 213).

La familia nuclear, fundada en el matrimonio, es "la primera estructura fundamental a favor de la ecología humana" (CA, n. 39). En ella aprendemos a comunicarnos y a respetar el medioambiente. Desafortunadamente, en nuestra sociedad numerosas familias marginan a los abuelos y evitan a los niños. Así, no es extraño que muchos se sientan "estrujados en el presente porque olvidan el pasado, y temen el futuro" (PASQUALE, 2017, p. 57) ${ }^{50}$.

\section{Conclusión}

En nuestra época se insiste más en "la familia relación" que en "la familia institución" y, en este sentido, se ha mejorado en muchos aspectos, como la libertad al momento de escoger la pareja, la igualdad entre los sexos y la gestión en común de las tareas domésticas. Sin embargo, la familia se ha vuelto también más inestable y sufre el consumismo e individualismo

50 "Una familia que no respeta y atiende a sus abuelos, que son su memoria viva, es una familia desintegrada" (AL, n. 193). 
que permea las relaciones sociales. La tecnología nos permite estar más conectados a nivel global, pero nuestras relaciones se han vuelto más superficiales.

En ese artículo, hemos visto que la familia es la "escuela más importante" (FRANCISCO, 2016b, p. 4) de espiritualidad y de comunicación en la lógica del don; es decir la "escuela privilegiada de generosidad, de compartir, de responsabilidad, escuela que educa a superar una cierta mentalidad individualista que se abrió camino en nuestras sociedades" (FRANCISCO, 2015c, p. 234). Resulta, por tanto, lamentable que la legislación de muchos países no ofrezca ventajas a la institución matrimonial sobre la simple convivencia ${ }^{51}$.

"La crisis de la familia dio origen a una crisis de ecología humana" 52 que afecta a la humanidad entera y a toda la creación, porque todo está relacionado. Se trata de una crisis de familia ${ }^{53}$; es decir, de una ruptura, a nivel global, de las relaciones gratuitas, fraternas y afectuosas que caracterizan a la vida familiar ${ }^{54}$.

El Papa Francisco invita a "hacer renacer entre todos un deseo mundial de hermandad" (FT, n. 8), de tal manera que podamos avanzar juntos hacia "un futuro plasmado por la interdependencia y la corresponsabilidad entre toda la familia humana" (FT, n. 127). Eso será posible si potenciamos la vida familiar, pues la experiencia de hermandad y la educación que se recibe en la familia tiene la capacidad de irradiar el estilo de la fraternidad "como una promesa sobre toda la sociedad" (AL, n. 194).

\footnotetext{
${ }^{51}$ Cf. DOMÍNGUEZ HIDALGO, C. Claves de política pública de familia planteadas por Amoris Laetitia: comentario al capítulo segundo "realidad y desafíos". Medellín, Bogotá, v. 165, p. 273-300, 2016. Aquí p. 286.

${ }^{52}$ FRANCISCO, Discurso en el coloquio internacional sobre la complementariedad del hombre y la mujer (17.11.2014), n. 2, en AAS 106/12 (2014) 979-981.

${ }^{53}$ Sobre el enfoque ecológico de la familia: ANDREWS, M. P.; BUBOLZ, M. M.; PAOLUCCI, B. An ecological approach to the study of the family. Marriage and family Review, Abingdon, v. 3, p. 29-49, 1980.

${ }^{54}$ CARB AJO NÚÑEZ, M. "Todo está conectado": Ecología integral y comunicación en la era digital. Lima: Paulinas, 2019. Sobre la distinción entre solidaridad y fraternidad: CARBAJO NÚNEZ, M. A free and fraternal economy: The Franciscan perspective. Delhi: Media House, 2018b. p. 105.
} 


\section{Siglas}
$\mathrm{AL}=$ Amoris laetitia
$\mathrm{CA}=$ Centesimus annus
$\mathrm{CV}=$ Caritas in veritate
$\mathrm{EG}=$ Evangelium gaudium
$\mathrm{FT}=$ Fratelli tutti
GS = Gaudium et spes
$\mathrm{PP}=$ Populorum progressio
LS = Laudato si' $^{\prime}$

\section{Referencias}

ADORNO, T. W.; HORKHEIMER, M. Dialectic of Enlightenment. New York: Herder \& Herder, 1997.

ANDREWS, M. P.; BUBOLZ, M. M.; PAOLUCCI, B. An ecological approach to the study of the family. Marriage and family Review, Abingdon, v. 3, p. 29-49, 1980.

AUGUSTINUS, De Trinitate. In: MIGNE, J.-P. (Ed.). Patrologia Latina. Paris, v. 42, p. $1841-1864$.

BENEDICTO XVI, Papa. Carta encíclica Deus caritas est. Acta Apostolicae Sedis, Vaticano, v. 98, n. 1, p. 217-252, 2006.

BENEDICTO XVI, Papa. Exhortación apostólica postsinodal Sacramentum Caritatis. Acta Apostolicae Sedis, Vaticano, v. 99, n. 3, p. 105-180, 2007.

BENEDICTO XVI, Papa. Discurso inaugural, Aparecida, 13.05.2007. In: CELAM. $V$ Conferencia General del Episcopado Latinoamericano y del Caribe, Documento conclusivo. Bogotá: Paulinas, 2008a.

BENEDICTO XVI, Papa. Meditación en la 12a Asamblea general ordinaria del Sínodo de los obispos, 6.10.2008. Ecclesia, n. 3436, p. 26, 2008 b.

BENEDICTO XVI, Papa. Carta encíclica Caritas in veritate. Acta Apostolicae Sedis, Vaticano, v. 101, n. 19, p. 641-709, 2009a.

BENEDICTO XVI, Papa. Audiencia general 02.12.2009. L'Osservatore Romano, Vaticano, p. 1, 03 dic. 2009b.

BENEDICTO XVI, Papa. Homilía en el VII Encuentro mundial de familias, 3.06.2012. In: BENEDICTO XVI, Papa. Insegnamenti di Benedetto XVI. Ciudad del Vaticano: LEV, 2012. v. VIII/1, p. 693-697.

BONFRATE, G.; YÁÑEZ, M. Amoris Laetitia, la Sapienza dell'amore: Fragilità e belleza della relazione nel matrimonio e nella famiglia. Roma: Studium, 2017.

BUENAVENTURA. Breviloquium, [Brevil.]. (Quaracchi V 230a).

CAPANTINI, L.; GRONCHI, M. La vulnerabilità. Cinisello Balsamo: San Paolo, 2018. 
CARBAJO NÚÑEZ, M. Generación híper-conectada y discernimiento: una perspectiva franciscana. Selecciones de Franciscanismo, Murcia, v. 138, p. 227-244, 2018a.

CARBAJO NÚÑEZ, M. A free and fraternal economy: the Franciscan perspective. Delhi: Media House, 2018b.

CARBAJO NÚÑEZ, M. “Todo está conectado": ecología integral y comunicación en la era digital. Lima: Paulinas, 2019.

CARBAJO NÚÑEZ, M. Education and integral ecology: the role of family, spirituality, and university. Carthaginensia, Murcia, v. 37, n. 71, 2021a.

CARBAJO NÚÑEZ, M. La fraternità nell'enciclica Fratelli tutti: Radici francescane. Collectanea Franciscana, Roma, v. 90, 2021b.

CARBAJO NÚÑEZ, M. Franziskanische Wirtschaft: Ein Vorschlag um aus der Krise Herauszukommen. Heiligenkreuz: Be\&Be Verlag, 2021c.

CATECISMO DE LA IGLESIA CATÓLICA. Ciudad del Vaticano: LEV, 1997

CID VÁZQUEZ, M. T., El amor como hilo conductor de la pastoral familiar en Amoris Laetitia. Familia, Salamanca, v. 54, p. 69-84, 2017.

CONCILIO VATICANO II. Constitución pastoral Gaudium et spes. Acta Apostolicae Sedis, Vaticano, v. 58, p. 1025-1120, 1966.

CONFERENCIA EPISCOPAL ESPAÑOLA. La familia, santuario de la vida y esperanza de la sociedad: Instrucción pastoral. Madrid: Palabra, 2001.

CONGREGACIÓN PARA LA EDUCACIÓN CATÓliCA. Pacto Educativo Global (PEG): Instrumentum laboris. Vaticano: 2020. Disponible em: <https://www.educationglobalcompact.org/it/ instrumentum-laboris/>. Acceso en: 14 oct. 2020.

COSTA, G. Il discernimento, cura delle famiglie nella Amoris Laetitia. Aggiornamenti sociali, Milano, v. 67, n. 5, p. 357-364, 2016.

DOMÍNGUEZ HIDALGO, C. Claves de política pública de familia planteadas por Amoris Laetitia: comentario al capítulo segundo "realidad y desafíos". Medellín, Bogotá, v. 165, p. 237-300, 2016.

FLORES, N. M. "Our sister, mother earth": solidarity and familial ecology in Laudato si'. Journal of Religious Ethics, New Jersey, v. 46, n. 3, p. 463-478, 2018.

FRANCISCO, Papa. Carta encíclica Lumen Fidei. Acta Apostolicae Sedis, Vaticano, v. 105, n. 43, p. 555-596, 2013 a.

FRANCISCO, Papa. Exhortación apostólica Evangelii gaudium. Acta Apostolicae Sedis, Vaticano, v. 105, n. 44, p. 1019-1137, 2013b.

FRANCISCO, Papa. Discurso en la sede de la FAO con motivo de la II Conferencia internacional sobre la nutrición, 20.11.2014. L'Osservatore Romano, Vaticano, n. 266, p. 8, 2014a.

FRANCISCO, Papa. Discurso en el coloquio internacional sobre la complementariedad del hombre y la mujer, 17.11.2014. Acta Apostolicae Sedis, Vaticano, v. 106, n. 12, p. 979-981, 2014 b.

FRANCISCO, Papa. Carta encíclica Laudato si'. Acta Apostolicae Sedis, Vaticano, v. 107, n. 9, p. 847-945, 2015a. 
FRANCISCO, Papa. Catequesis sobre la familia, 16.09.2015. L'Osservatore Romano, Vaticano, n. 211, p. 8, 17 sept. 2015 b.

FRANCISCO, Papa. Mensaje a los participantes en la 47 Semana social de los católicos italianos (11.09.2013). In: Insegnamenti di Giovanni Paolo II. Ciudad de Vaticano: LEV, 2015c. v. I/2, p. 231-234.

FRANCISCO, Papa. Exhortación apostólica postsinodal Amoris Laetitia. Acta Apostolicae Sedis, Vaticano, v. 108, n. 4, p. 311-446, 2016 a.

FRANCISCO, Papa. Discurso al cuerpo diplomático acreditado ante la Santa Sede, 11.01.2016. L'Osservatore Romano, Vaticano, n. 156/7, p. 4-5, 11/12 ene. 2016b.

FRANCISCO, Papa. Constitución Apostólica Veritatis gaudium. Regno-Documenti, Vaticano, n. 63, n. 5, p. 137-165, 2018a.

FRANCISCO, Papa. Exhortación apostólica Gaudete et exsultate. Ciudad del Vaticano: LEV, 2018b.

FRANCISCO, Papa. Discurso a los fieles en Pietrelcina, 17.03.2018. L'Osservatore Romano, Vaticano, v. 64, 18 mar. 2018c.

FRANCISCO, Papa. Audiencia general, 18.03.2015. L'Osservatore Romano, Vaticano, n. 64, 19 mar. $2018 \mathrm{~d}$.

FRANCISCO, Papa. Mensaje para el lanzamiento del pacto educativo, 12.09.2019. L'Osservatore Romano, Vaticano, n. 207, p. 8, 13 sept. 2019.

FRANCISCO, Papa. Carta encíclica Fratelli tutti. Ciudad de Vaticano: LEV, 2020a.

FRANCISCO, Papa. Discurso al cuerpo diplomático acreditado ante la Santa Sede, 9.01.2020. L'Osservatore Romano, Vaticano, n. 6, p. 4-5, 10 ene. 2020b.

HOBBES, T. The Leviathan. New York: Crooke, 1651.

JUAN PABLO II, Papa. Homilía en Puebla de los Ángeles, 28.01.1979. In: JUAN PABLO II, Papa. Insegnamenti di Giovanni Paolo II. Ciudad del Vaticano: LEV, 1979. p. $180-185$.

JUAN PABLO II, Papa. Carta encíclica Centesimus annus. Acta Apostolicae Sedis, Vaticano, v. 83, p. 796-867, 1981.

JUAN PABLO II, Papa. Exhortación apostólica Familiaris consortio. Acta Apostolicae Sedis, Vaticano, v. 74, p. 81-191, 1982.

JUAN PABLO II, Papa. Carta a las familias Gratissimam sane. Acta Apostolicae Sedis, Vaticano, v. 86, p. 868-925, 1994a.

JUAN PABLO II, Papa. Cruzando el umbral de la esperanza. Barcelona: Plaza \& Janés, 1994b.

JUAN PABLO II, Papa. Ángelus 30.01.1994. In: JUAN PABLO II, Papa. Insegnamenti di Giovanni Paolo II. Ciudad de Vaticano: LEV, 1996. v. XVII/1, p. 231-234.

KASPER, W. El evangelio de la familia. Santander: Sal Terrae, 2014.

KONRATH, S.; O'BRIEN E.H.; HSING C. Changes in Dispositional Empathy in American College Students over Time: A Meta-analysis. Personality and Social Psychology Review, Thousand Oaks, v. 15, n. 2, p. 180-198, 2011. 
LADARIA, L. F. La Trinidad, Misterio de Comunión. Salamanca: Secretariado Trinitario, 2002.

MAJORANO, S. The Alphonsian criteria of merciful pastoral discernment in the light of chapter VIII of the exhortation Amoris Laetitia. Studia Moralia, Roma, v. 55, n. 1, p. 7-32, 2017.

MCFAGUE, S. The body of God: An ecological theology. Minneapolis: Fortress, 1993. MIGNE, J.-P. (Ed.). Patrologia Latina. Paris: 1841-1864. 226 v.

PABLO VI, Papa. Carta encíclica Populorum progressio. Acta Apostolicae Sedis, Vaticano, v. 59, p. 257-299, 1967.

PASQUALE, G. Sensus fidei: luogo privilegiato del discernimento. CredereOggi, Padova, v. 221, p. 55-69, 2017.

SALONIA, G. Amoris Laetitia: Dalla sapienza evangelica percorsi di crescita nelle relazioni familiari. In: BONFRATE, G.; YÁÑEZ, H. M. (Ed.). Amoris Laetitia, la sapienza dell'amore: Fragilità e bellezza della relazione nel matrimonio e nella famiglia. Roma: Studium, 2017. p. 168-182.

SPADARO, A.; CAMELI, L. J. La sfida del discernimento in Amoris Laetitia. La Civiltà Cattolica, Roma, v. 3985, n. 3, p. 3-16, 2016.

TEJERO, E. El evangelio de la casa y de la familia. Pamplona: Eunsa, 2014.

Artículo sometido en 04.01.2021 y aprobado en 07.04.2021.

Martín Carbajo Núñez, OFM Doctor en teología moral (Academia Alfonsiana, Roma), una licenciatura en filología germánica (Universidad de Santiago de Compostela). Profesor extraordinario en la Pontificia universidad Antonianum. Orcid.org/0000-0002-2814-5688. E-mail: mcarbajo@sandiego.edu

Dirección: Collegio internazionale Sant'Antonio

PP. Francescani

Via Merulana 124

0185 Roma - Italia 